Methods The study consisted of 2886 SLE patients, including 947 (33\%) with LN. The discovery cohort (Sweden, $\mathrm{n}=1091$ ) and replication cohort 1 (US, $\mathrm{n}=962$ ) were genotyped on the Immunochip and replication cohort 2 (Norway/Denmark, $n=833$ ) on a custom array chip. Allele frequencies were compared between patients with $\mathrm{LN}$ and LN-negative patients. SNPs with a p-value $<0.001$ in the $\mathrm{LN}$ vs LN-negative analysis in the discovery cohort $\left(\mathrm{n}_{\mathrm{SNPs}}=139\right)$ were analyzed in replication cohort 1 . Ten SNPs associated to LN $(\mathrm{p}<0.0002)$ in the discovery cohort were genotyped in replication cohort 2. A Bonferroni-corrected $p$-value of $<1.0 \times 10^{-6}$ correcting for 48,000 independent SNPs was considered significant.

Results In the discovery cohort, strong association to $\mathrm{LN}$ was found with several highly linked SNPs in BANK1, with the top signal in the intronic SNP rs4699261 $\left(\mathrm{p}=9.9 \times 10^{-5}\right.$, OR 0.66$)$. The association was also present in replication cohort $1\left(p=9.5 \times 10^{-4}\right)$. In a meta-analysis of the discovery and replication cohort 1 , a total of 20 SNPs in BANK1 were associated to LN with the highest signal in rs4699261 $\left(\mathrm{p}=3.3 \times 10^{-7}\right)$. There was a tendency towards association in replication cohort $2(\mathrm{p}=0.05)$ and in a meta-analysis of all cohorts, the association with BANK1 was strengthened $\left(\mathrm{p}=1.7 \times 10^{-7}\right)$.

Conclusion BANK1 variations are associated with $\mathrm{LN}$ in patients with SLE. Upregulated BANK1 expression in renal biopsies from LN patients has previously been shown, however the exact role of BANK1 in LN pathogenesis remains to be elucidated.

\section{B-CELL DEPLETION AND RESPONSE IN A RANDOMIZED, CONTROLLED TRIAL OF OBINUTUZUMAB FOR PROLIFERATIVE LUPUS NEPHRITIS}

${ }^{1}$ Richard Furie, ${ }^{2}$ Matthew D Cascino, ${ }^{2}$ Jay P Garg, ${ }^{3}$ Gustavo Aroca, ${ }^{4}$ Analía Alvarez, ${ }^{5}$ Hilda Fragoso-Loyo, ${ }^{6}$ Elizabeth Zuta Santillán, ${ }^{7}$ Ana Malvar, ${ }^{2}$ Paul G Brunetta, ${ }^{8}$ Thomas Schindler, ${ }^{2}$ Cary Michael Donna Looney, ${ }^{9}$ Imran Hassan, ${ }^{10}$ Brad H Rovin. ${ }^{1}$ Northwell Health Great Neck, New York; ${ }^{2}$ Genentech, Inc., South San Francisco, USA; ${ }^{3}$ Simon Bolivar University y Clinica de la Costa, Barranquilla, Colombia; ${ }^{4}$ CEMIC, Buenos Aires, Argentina; ${ }^{5}$ Instituto Nacional de Ciencias Médicas y Nutrición Salvador Zubirán, Mexico City, Mexico; ${ }^{6}$ Instituto de Ginecología y Reproducción, Lima, Peru; ${ }^{7}$ Organización Maedica de Investigación, Buenos Aries, Argentina; ${ }^{8} \mathrm{~F}$. Hoffmann-La Roche AG, Basel, Switzerland; ${ }^{9}$ Hoffmann-La Roche Ltd, Mississauga, Canada; ${ }^{10}$ Ohio State University, Columbus, USA

\subsection{6/lupus-2020-eurolupus.46}

Background Previous analyses identified associations between the degree of B-cell depletion and response in lupus nephritis (LN). NOBILITY tested whether enhanced B-cell depletion with the type II anti-CD20 mAb obinutuzumab could improve responses in $\mathrm{LN}$.

Methods 126 patients with active Class III/IV LN were randomized to obinutuzumab or placebo infusions in combination with mycophenolate and corticosteroids. Peripheral B-cells were measured using a flow cytometry method with a lower limit of 0.441 cells $/ \mu \mathrm{L}$. Sustained depletion was assessed by flow cytometry measurements at weeks 24 and 52 .

Results Obinutuzumab was associated with increased CRR $(40 \%$ vs. $18 \%, \mathrm{P}=0.007)$ and $\mathrm{ORR}(51 \%$ vs. $29 \%, \mathrm{P}$ $=0.015)$ at week 76. Obinutuzumab resulted in rapid and

Abstract 035 Table 1 Renal responses at week 76 by depletion status

\begin{tabular}{|l|c|c|c|}
\hline & $\begin{array}{c}\text { Obinutuzumab } \\
\text { sustained depletion } \\
(\mathbf{N}=\mathbf{3 2})\end{array}$ & $\begin{array}{c}\text { Obinutuzumab } \\
\text { detectable B-cells } \\
(\mathbf{N}=\mathbf{2 0})\end{array}$ & $\begin{array}{c}\text { Placebo group } \\
(\mathbf{N}=62)\end{array}$ \\
\hline CRR & $50 \%^{* *}$ & $35 \%^{*}$ & $18 \%$ \\
\hline Modified CRR & $7 \%^{* *}$ & $50 \%$ & $37 \%$ \\
\hline ORR & $66 \%^{* * *}$ & $45 \%^{*}$ & $29 \%$ \\
\hline
\end{tabular}

Eleven patients in the obinutuzumab group with insufficient data to determine depletion status are excluded.

${ }^{*} P<0.2$ vs. placebo group
${ }^{* *} P<0.05$ vs. placebo group
${ }^{* * *} P<0.001$ vs. placebo group

$\mathrm{CRR}=$ complete renal response, which required UPCR $<0.5$ with serum creatinine $\leq$ the upper limit of normal and not increased $>15 \%$ from baseline with $<10$ RBCs/HPF and no RBC casts.

Modified CRR $=$ UPCR $<0.5$ with normal serum creatinine.

ORR = overall renal response, which required either CRR or partial renal response: $\geq 50 \%$ reduction in UPCR from baseline to $<1$ ( $<3$ if baseline UPCR $\geq 3$ ) with serum creatinine not increased $>15 \%$ from baseline and $\leq 50 \%$ increase in urinary RBCs (or $<10 \mathrm{RBCs} / \mathrm{HPF}$ ). 
complete depletion of peripheral B-cells, memory and naïve Bcell subsets, and plasmablasts, with $89 \%$ of obinutuzumab patients depleted to $<0.441 \mathrm{CD} 19+$ cells $/ \mu \mathrm{L}$ at week 4 . Among obinutuzumab patients, sustained B-cell depletion was associated with greater renal response at week 76 (table 1), although patients who achieved sustained depletion also had lower baseline proteinuria and serum creatinine.

Conclusions Obinutuzumab, a type II anti-CD20 mAb, induced rapid and complete depletion of peripheral B-cells and B-cell subsets. Similar to previous reports, sustained Bcell depletion was associated with increased renal response. Further evaluation is ongoing to understand the factors associated with achievement of sustained B-cell depletion and renal response.

Acknowledgements This study was funded by F. Hoffmann-La Roche.

\section{LOW-DOSE IL-2 THERAPY MODULATES LYMPHOCYTE SUBSETS THAT ARE INVOLVED IN THE REGULATION OF GERMINAL-CENTRE REACTIONS IN PATIENTS WITH SLE}

${ }^{1,2}$ Jens Y Humrich, ${ }^{1}$ Caroline von Spee-Mayer, ${ }^{1}$ Elise Siegert, ${ }^{1}$ Martina Bertolo,
${ }^{1}$ Angelika Rose, ${ }^{1}$ Philipp Enghard, ${ }^{1}$ Falk Hiepe, ${ }^{1}$ Tobias Alexander, ${ }^{1}$ Gerd-
Rüdiger Burmester, ${ }^{1,2}$ Gabriela Riemekasten. ${ }^{1}$ Dept. of Rheumatology and Clinical
Immunology, Charité - University Medicine Berlin, Berlin; ${ }^{2}$ Dept. of Rheumatology and
Clinical Immunology, University Hospital Schleswig-Holstein - Campus Lübeck, Lübeck,
Germany

10.1136/lupus-2020-eurolupus. 47

Background Low-dose IL-2 therapy was shown to restore regulatory $\mathrm{T}$ cell (Treg) homeostasis and to decrease disease activity in early phase clinical trials in patients with SLE. ${ }^{1,2,3}$ However, the mechanisms of action of IL-2 therapy beyond Treg targeting are still poorly understood. Here, we assessed the changes of lymphocyte subsets in the peripheral blood that are considered to be involved in the regulation of germinal-center reactions during an open-label, uncontrolled, phase $1 / 2$ a single-center trial with low-dose IL-2 therapy in patients with active SLE.

Methods 12 patients with active and refractory SLE (SLEDAI $\geq 6$ ) were treated with four separate 5-day treatment cycles consisting of daily subcutaneous injections of IL-2 between 0.75 and 3.0 million IU. Cells from peripheral blood were analyzed by flow cytometry before and one day after each treatment cycle.

Results Significant decreases in the numbers of CD3-CD56CD19+ B cells were observed after most treatment cycles and at the end of the treatment period. Subtyping of the B cell population revealed that in particular proportions and numbers of $\mathrm{CD} 20+\mathrm{IgD}+\mathrm{CD} 27+$ marginal-zone $\mathrm{B}$ cells were reduced. Transient and moderate decreases in the proportions and numbers of CD20+IgD-CD27- memory B cells were also apparent during the treatment cycles, whereas there were no relevant changes in $C D 20+\operatorname{IgD}+\mathrm{CD} 27$ - naïve $\mathrm{B}$ cells. In addition we noted moderate decreases in the proportions of common CXCR5 $+\mathrm{T}$ follicular helper cells (Tfh) and of CD45RO+CCR7-CXCR5+PD1+ Tfh cells among $\mathrm{CD} 3+\mathrm{CD} 4+$ FoxP3 $-\mathrm{T}$ cells during the treatment period. Yet, more pronounced decreases were observed in the proportions of CXCR5+ cells among CD3+CD4 + FoxP3 + regulatory $\mathrm{T}$ cells.
Conclusions These findings suggest that low-dose IL-2 therapy interferes with the regulation and fine tuning of immune responses that take place in the germinal-centres of lymphatic tissues.

Funding German Research Foundation (DFG).

\section{REFERENCES}

1. Humrich JY, von Spee-Mayer C, Siegert E, Alexander T, Hiepe F, Radbruch A, Burmester GR, Riemekasten G. Rapid induction of clinical remission by lowdose interleukin-2 in a patient with refractory SLE. Ann Rheum Dis. 2015; 74; 791-792.

2. von Spee-Mayer $C$, Siegert E, Abdirama D, Rose A, Klaus A, Alexander T, Enghard P, Sawitzki B, Hiepe F, Radbruch A, et al. Low-dose interleukin-2 selectively corrects regulatory $T$ cell defects in patients with systemic lupus erythematosus. Ann Rheum Dis. 2016; 75; 1407-1415.

3. Humrich JY, von Spee-Mayer $C$, Siegert $E$, Bertolo $M$, Rose $A$, Abdirama $D$, Enghard $P$, Stuhlmüller B, Sawitzki B, Huscher D, et al. Low-dose interleukin-2 therapy in refractory systemic lupus erythematosus: an investigator-initiated, single-centre phase 1 and 2a clinical trial. Lancet Rheumatol. 2019; 1; e44-e54.

\section{M5049, A NOVEL POTENT AND SELECTIVE INHIBITOR OF TOLL-LIKE RECEPTORS 7 AND 8 (TLR 7/8)}

${ }^{1}$ Brian Sherer, ${ }^{2}$ Andrew T Bender, ${ }^{2}$ Albertina Pereira, ${ }^{3}$ Sonja Reissig, ${ }^{3}$ Philipp Haselmayer, ${ }^{2}$ Shinji L Okitsu, ${ }^{2}$ Evgeni Tzvetkov, ${ }^{2}$ Melinda Przetak, ${ }^{2}$ Noune Tahmassian Morse, ${ }^{2}$ Jaromir Vlach. 'US Chemistry, EMD Serono*, Billerica; ${ }^{2}$ TIP Immunology, EMD Serono*, Billerica, USA; ${ }^{3} T I P$ Immunology, Merck Healthcare KGaA, Darmstadt, Germany; *A business of Merck KGA, Darmstadt, Germany

\subsection{6/lupus-2020-eurolupus.48}

Background TLR 7/8 are transmembrane receptors that recognize single-stranded RNA. In humans, TLR7 is mainly expressed in B cells and plasmacytoid dendritic cells and its activation stimulates antibody production and secretion of cytokines including type I interferon (IFN). Activation of TLR8 in myeloid cells leads to cytokine production, and activation of antibacterial host-protective mechanisms (eg, neutrophil NETosis). Aberrant activation of TLR $7 / 8$ is potentially pathogenic and linked to progression of certain autoimmune diseases such as SLE. Thus, inhibition of TLR7/8 may be an effective treatment approach for SLE.

Methods Compounds were prepared and optimized for potency by testing them in TLR7/8-stimulated HEK 293 cells, peripheral blood mononuclear cells (PBMCs), and human whole blood. M5049 was selected as the compound with the best balance of potency, pharmacokinetic, and physiochemical properties. The pharmacokinetic and pharmacodynamic activity of M5049 was assessed in mice to determine its in vivo potency and duration of action. M5049 was also tested in mouse lupus models.

Results M5049 showed potent and selective activity in a wide range of cellular assays for inhibition of TLR7/8. M5049 inhibited R848-stimulated cytokine production in human PBMCs and whole blood, and TLR8-mediated NETosis in human primary neutrophils. In mice, M5049 showed a dose-dependent inhibition of R848-stimulated cytokine release and a long duration of action. M5049 demonstrated efficacy in suppressing disease development in the BXSB-Yaa and IFN- $\alpha$ accelerated NZB/W F1 mouse models of lupus.

Conclusions M5049 is a highly potent and selective inhibitor of TLR 7/8 that effectively reduces lupus pathogenesis in mice. M5049 may have potential to treat autoimmune diseases such as SLE in humans. 